\title{
Über die sogenannte sowjetestnische Literatur
}

\author{
JAAN UNDUSK
}

\begin{abstract}
Of the So-Called Soviet Estonian literature. The aim of Socialist Realism was not to define aesthetically the new Socialist art, but rather to provide a tool for ideological control. It contained a deliberate contradiction. This contradiction was called dialectical, and it seemingly had to guarantee the flexibility of the method. The artist had to connect the depiction of the harsh reality of life with the bright hopes of tomorrow, class struggle with the peacefulness of a classless society. Actually, this "dialectic" gave a chance only to those who participated in various purges directed against those who did not conform. These purges, or campaigns of purification, were always "dialectical": against "formalism and naturalism", or against "cosmopolitanism and nationalism". One could attack almost every literary phenomenon under these flags, and during the campaigns, every author had every reason to be scared of being accused - which actually was the great idea of them.
\end{abstract}

Keywords: Soviet literature, Estonian literature in exile, the Stalinist purges, dialectic

DOI: http://dx.doi.org/10.12697/IL.2013.18.2.15

Lassen Sie mich zu Beginn eine ganz allgemeine Frage stellen: Was für ein Phänomen war die sogenannte sowjetische (darunter auch die sowjetestnische) Literatur in Zeit und Raum? Oder, um die Aufgabe in kantianischen Begriffen zu formulieren: Wie steht es eigentlich mit den apriorischen Formen der Anschauung, dem Raum und der Zeit, durch die wir gewohnt sind, die sowjetische Kultur im Baltikum zu betrachten?

Man spricht zum Beispiel über die estnische Literatur der 1860er Jahre und man spricht über die estnische Literatur der 1930er Jahre. Man kann die estnischsprachige Literatur von ihren Anfängen bis zum Ende des Zweiten Weltkriegs 1945 unterschiedlich periodisieren. Dabei ist es fast sicher, dass man die ganze estnischsprachige Literatur erfasst. Die estnische Literatur vor 1945 ist einheitlich periodisierbar. Und nicht etwa deswegen, weil diese Literatur jahrhundertelang nur auf dem estnischen Gebiet entstanden wäre. So war es auch nicht. Es gab z. B. politisch exilierte estnische oder lettische Schriftsteller nach der russischen Revolution von 1905 (Eduard Vilde, Friedebert Tuglas, 
Jānis Rainis), die in Finnland oder Dänemark, in der Schweiz oder in den USA lebten, die aber physisch mit ihren Werken und geistig durch die Rezeption in der Heimat anwesend waren. Ungeachtet auch ideologischer oder ästhetischer Kontraste und Auseinandersetzungen hat sich die Literatur in einem kulturellen Kontext niedergeschlagen und auf der Basis eines einzigen kommunikativen Gedächtnisses funktioniert.

Nach dem Zweiten Weltkrieg wurde das anders. Ich erlaube mir zu sagen, dass es bis heute keine verallgemeinernde Geschichte der estnischen Literatur der 1940er Jahre gibt. Es gibt auch keine solche für die Literatur der 1950er oder sogar der 1960er Jahre. Warum behaupte ich etwas Derartiges, obwohl über die Literatur und die Autoren dieser Jahrzehnte viel geschrieben worden ist? Weil für die Jahrzehnte, als das Gebiet Estlands von der Sowjetmacht okkupiert, dabei ein großer Teil der Kultur aus Estland verbannt und ins Ausland geflossen war, eine durchgängige zeitliche Gliederung der estnischsprachigen Literatur normalerweise der erste Schritt der Analyse - einfach nicht stattgefunden hat. Man beginnt bei der Analyse des Literaturprozesses nicht mit einem zeitlichen Nacheinander, sondern mit einem räumlichen Nebeneinander: Zuerst trennt man Estland und Nicht-Estland (oder die sogenannte übrige Welt), den Okkupationsraum und den Exilraum. Wenn auch nicht explizit, dann doch implizit (z. B. bei der Behandlung einzelner Autoren, wo man die Bemerkung „seit 1944 im Exil” nie auslassen kann). Und erst dann kommt die Zeit ins Spiel und man beginnt zu periodisieren, in zwei Räumen getrennt. Obwohl diese „übrige Welt” (Westeuropa, Amerika, Australien), d. h. Nicht-Estland oder Außer-Estland, geographisch unermesslich größer ist als das kleine Gebiet Estland, ist sie ein Raum und Estland der andere Raum der estnischsprachigen Literatur. Toronto und Sydney sind einander in diesem Weltbild unvergleichbar näher als Stockholm und Tallinn. Und diese Priorität der räumlichen Einteilung vor der zeitlichen konnte man bisher nicht vermeiden, woraus auch meine erste These folgt, nämlich, dass der Begriff „die sowjetestnische Literatur” in erster Linie eine räumliche Definition der Literatur darstellt - wie auch ihr Antipode, die Exilliteratur. Man will damit zeigen, dass sie beide als geopolitisch getrennte Erscheinungen, als ganzheitliche ästhetische Räume zu verstehen sind, und nicht einfach als zwei (feindliche) ideologische Strömungen. Wer „sowjetestnisch” sagt, muss an den Lebensraum denken. Anderenfalls läuft man Gefahr, in allem „Sowjetischen” bloß eine ideologische Perversion zu erblicken. Es gab Stimmen sowohl im Exil als auch Ende der 1980er Jahre in Estland, die forderten, die ganze in Sowjetestland entstandene Literatur über Bord zu werfen. Das war die logische Folgerung aus der These, dass die estnische Literatur zur Zeit der sowjetischen Okkupation nur eine nationale oder ideologische Anomalie, nur eine Fortsetzung der eindeutig politisierten 
sowjetischen - und nicht der estnischen - Literatur war, und keinen ernst zu nehmenden kulturellen Raum bildete. Die sowjetestnische Literatur war in diesem Fall nur eine Randerscheinung der sowjetischen Literatur auf Estnisch.

Warum ist es wichtig, darüber zu sprechen? Weil hinter der uneinheitlichen Periodisierung der estnischen Literatur dieser Epoche sich doch ein Symptom versteckt. Ein Symptom dafür, dass etwas doch falsch gewesen ist, was die Umschaltung von Zeit auf Raum - als den ersten Gliederungsfaktor erfordert. Man lebt in Estland schon mehr als zwanzig Jahre unter der Losung „Die estnische Literatur ist eins“, das heißt, es gibt nur eine einzige estnischsprachige Literatur mit ihrem einzig wirklichen Existenzraum, der die Sprache selbst ist. Alle Literatur auf Estnisch gehört zur estnischen Literatur, der Entstehungsort ist nicht wichtig. Nun aber beginnt man über die Dichtung der 1950er Jahre zu reflektieren und spricht sogleich eine erklärende - oder entschuldigende? - Warnung aus: Es gab den Okkupationsraum und das Exil, das heißt zwei literarische Räume in einem einzigen sprachlichen Raum. Ohne diese Klausel wagt man nicht weiterzugehen. In groben Umrissen findet sich dasselbe Symptom wahrscheinlich ganz ähnlich in zahlreichen anderen osteuropäischen Literaturen.

Eine etwas blauäugige Kulturliebhaberin aus dem Westen hat mich einmal gefragt: Wie kam es, dass zwei estnische Schriftsteller, Juhan Smuul und Ilmar Laaban, die gleichaltrig und gleich begabt waren, Ende der 1940er Jahre so unterschiedliche Gedichte geschrieben haben, der erste seine naiven Hymnen auf Jossif Stalin, der andere geistreich-surreale, anarchistische, fast antikommunistische Texte? Die ganze Situation war nicht als eine normale Widerspiegelung demokratischer Mannigfaltigkeit $\mathrm{zu}$ verstehen. Ich musste sie daran erinnern, dass der erste Mann in Tallinn, der andere in Stockholm tätig war. Nun habe ich große Augen gesehen: Musste man in Tallinn damals unbedingt dumme Gedichte schreiben? Warum ist er dann eigentlich nicht nach Stockholm gefahren? Und so weiter. So kommt man immer wieder dazu, über die Okkupation und die Emigration und die zwei literarischen Räume zu reden. Und die Sache kann noch komplizierter sein. Derselbe Ilmar Laaban, der 1944 in Stockholm als erster estnischer Theoretiker des Surrealismus auftrat, ist ja drei Jahre zuvor in Tallinn einer der ersten estnischen Theoretiker des Sozialistischen Realismus gewesen. 1941 beschimpfte Laaban die „trotzkistischen Verräter“, die sich als Kommunisten tarnen (Laaban 1941: 162), 1944 war André Breton, der wichtigste surrealistische Ideologe und ein Anhänger Trotzkis, sein Vorbild und er selbst ein eindeutiger Gegner des Stalinismus geworden (vgl. Laaban 1997). Es gab natürliche Übergänge zwischen den zwei Realismen auch im internationalen Maßstab, beide Ideologien waren ja links orientiert und verherrlichten das Prinzip der Dialektik. 
Die führenden französischen Surrealisten sind 1927 in die Kommunistische Partei Frankreichs eingetreten (Breton und Paul Éluard waren zwar 1932 schon ausgewiesen), Breton hat anfangs im Sozialistischen Realismus eine neue humanistische Perspektive gesehen (später denunzierte er diese „Methode” öffentlich), und aus Louis Aragon ist ein Romancier geworden, der bewusst den Prinzipien des Sozialistischen Realismus zu folgen versuchte. Doch ist damit die rasche Wende in den poetologischen Einstellungen Laabans noch nicht hinreichend erklärt. Wieder braucht man Hintergrundwissen, um die ganze Geschichte zu verstehen: Laaban ist inzwischen mit dem Schiff von Tallinn nach Stockholm gefahren (ausführlicher darüber siehe Undusk 2008: 23f., Undusk 2013: 57-61).

Aber muss man das eigentlich wissen? Muss man denn unbedingt von zwei literarischen Räumen reden, um die Literatur dieser Jahre verstehen zu können?

Einerseits gab es wirklich zwei getrennte Räume. Nicht nur, dass die meisten Autoren, die nach dem Zweiten Weltkrieg ins Exil gegangen waren (und diese waren die Mehrheit der führenden Schriftsteller) oder erst dort Autoren wurden, in der Heimat tabuisiert waren, sondern - und das ist viel wichtiger - dass ihre Werke aus der Heimat verbannt waren: Man konnte sie in Estland öffentlich weder erwerben noch rezensieren, man las und besprach sie fragmentarisch und nur in kleineren Kreisen. Die früheren Werke der älteren Autoren waren nicht selten in den sogenannten Spezialbeständen der Bibliotheken eingeschlossen. So hat sich die Situation prinzipiell verändert, wenn man sie mit dem politischen Exil nach der Revolution von 1905 vergleicht: Wie gesagt, damals lebten zwar einige Autoren im Exil, ihre Werke konnte man aber in der Heimat veröffentlichen und sie wurden auch allgemein gelesen. Nicht die Texte, nicht die literarische Produktion - nur die Menschen, die Autoren der Texte waren damals exiliert. Nicht mehr so nach dem Zweiten Weltkrieg. Wenn ein estnischer Autor schon in Stockholm oder Toronto lebte, dann existierten seine Werke für die Leser in Tallinn oder Tartu öffentlich nicht mehr und sie fielen einfach aus dem Literaturprozess heraus.

Dabei konnten die Werke der Exilautoren doch auch nach dem Zweiten Weltkrieg als ästhetische Geheimware in der Heimat höchst wirksam sein. Der Roman von Karl Ristikivi, einer der wichtigsten der estnischen Literatur des 20. Jahrhunderts, Hingede öö (Die Nacht der Seelen), ist im Todesjahr Stalins (1953) in Schweden erschienen und hat trotz seiner modernistischen Erzähltechnik und existentialistischen Grunderfahrung auch beim breiten Publikum im Exil einen enormen Erfolg gehabt. Im okkupierten Estland las man das Buch in privaten Kreisen erst Jahre später. Doch hat der stille Einfluss auf andere Dichter funktioniert: Die neue Prosa der 1960-1970er Jahre wurde 
im okkupierten Estland nicht nur unter dem Zeichen von Kafka, Hemingway oder sogar Arthur Schnitzler (der innere Monolog bei Jaan Kross!) geboren, sondern hat seine Wurzeln auch im Roman von Ristikivi. 1972 veröffentlichte der kommunistische Schriftsteller Paul Kuusberg in Estland seinen ebenso guten Roman Üks öö (Eine Nacht), der einen staatlichen Preis errang. Weder die Verantwortlichen auf der offiziellen Seite noch das breite Publikum ahnten wahrscheinlich die polemische Zusammengehörigkeit der Romane von Ristikivi und Kuusberg: Der erste stellt beängstigende Fragen an das westliche, der andere an das östliche Exil der Esten (im sowjetischen Hinterland) während des Zweiten Weltkriegs. Vor uns liegt ein gutes - und keinesfalls das einzige - Beispiel für den verheimlichten Stoffwechsel zwischen den offiziell getrennten Kulturräumen.

Doch ist das alles nicht genug, um damit die heutigen Vorurteile zu begründen.

Hinter der Konzeption der zwei geopolitisch getrennten Räume in einer Sprache steht die Meinung, dass es zu gewissen Zeiten zwei estnische Sprachen gab: eine richtige und eine falsche. Die Konzeption der Räume ist ein diplomatischer Ausdruck dieser Erkenntnis. Die offizielle Ideologie der Sowjetzeit hat, aufgrund der leninistischen These, wonach in jeder vorsozialistischen Kultur zugleich zwei Kulturen, die bürgerliche und die demokratische, vorhanden seien, behauptet, dass die in der Emigration die falsche Sprache war. Die kulturpolitischen Stimmen im Exil - besonders während der ersten Jahrzehnte der Okkupation - haben das Gegenteil verkündet: Die falsche Sprache, oder wenigstens die Elemente derselben, sei eben in dem Textkorpus zu finden, der auf dem Gebiet Estlands unter der Sowjetmacht zustande gekommen sei. Nach der Wende haben wir uns - offiziell und inoffiziell - fast einstimmig diese letztgenannte politische Rhetorik angeeignet. Falsche Elemente hat die Sprache im Okkupationsraum beinhaltet, weil sie dort politisch erzwungen war. Natürlich nimmt man an, dass der Anteil der falschen Sprache bei verschiedenen Autoren und in verschiedenen Zeiten gewechselt hat: Die stalinistischen Lobgedichte oder Ideendramen haben von der falschen Sprache reichlich Gebrauch gemacht, aber beginnend mit dem politischen Tauwetter ist der Anteil der offenen Falschheit geringer geworden, und es gab kluge Autoren, die sie weitgehend umgehen konnten, durch die geschickte Wahl der Stoffe (z. B. aus der Geschichte), abstrakte, symbolische, allegorische Sujets und Handlungsweisen, karnevalistische Charaktere, metaphorische Sprache usw. Natürlich litt darunter eine echte direkte Wiedergabe der brennenden Probleme von Geschichte und Gegenwart. Tatsache ist, dass die meisten künstlerischen Hochleistungen der estnischen Literatur (die Jahre des offenen staatlichen Terrorismus ausgenommen) 
UNDUSK

letzten Endes im sowjetischen Raum und nicht im Exil zur Welt gekommen sind: Hier wuchsen die neuen Generationen, dort starben die alten aus. Und dies kam zum Teil - paradoxerweise! - auch daher, dass die Sprache der Kunst in der okkupierten Heimat komplizierter, feiner oder barocker wurde, politisch mehr schwieg, Andeutungen und halbdunkle Analogien bot, als sie öffentlich bekannt machte. Die Kunst entwickelte sich auf Kosten des Realitätsgefühls. Ich erinnere mich, wie platt mir manchmal die politisch unverhüllte Sprache der Exilromane erschien - wie Journalismus. Andererseits kann man aber auch das künstlerisch hochkarätige Spiel an der Grenze des Schweigens als politische Falschheit qualifizieren.

Wie dem auch sei: Eben deshalb, weil wir dem Gefühl der Falschheit und Schuld im Zusammenhang mit der Sowjetzeit nicht entkommen, sprechen wir über die sowjetestnische und nicht einfach über die estnische Literatur dieser Zeit. Und eben deshalb, weil wir die Autoren beschützen wollen, die existenziell nicht schuld daran waren, dass sie durch ihre Geburt in das Sowjetsystem geworfen waren und nicht von zu Hause weglaufen konnten oder wollten, verstehen wir das Adjektiv „sowjetisch” räumlich, als ein Attribut eines bestimmten geopolitischen Raumes, wo die Sprache mehr oder weniger von erzwungener Art war.

Daraus ergeben sich aber weitere kulturphilosophische Fragen. Die „Falschheit” der Sprache in der obigen Deutung ist politisch bestimmt. Aber eine künstlerische Leistung - wenn sie eine solche ist-sollte im Prinzip politisch nicht kontrollierbar sein. Antike, mittelalterliche, neuzeitliche Autoren haben künstlerisch hervorragende Hymnen auf gewalttätige Herrscher geschrieben, warum also nicht auch auf Jossif Stalin? Oder auf Adolf Hitler? Der Name, sei er Caesar, Napoleon oder Hitler, geht vorüber, das hohe Wortkunstwerk bleibt ungeachtet dessen. Doch wenn man fragt, ob die heutige deutsche Gesellschaft bereit wäre, in einem künstlerisch tadellosen Gedicht zu Ehren Adolf Hitlers eine ästhetische Leistung zu erkennen, muss man wahrscheinlich mit einem klaren „Nein” antworten. Das Wort „Hitler” vertritt in diesem Kontext eine falsche Sprache. Ein einziges Wort kann die mächtige poetische Struktur zerstören - ist es wirklich so? Wenn man in demselben Gedicht anstelle von „Hitler” einen Namen wie „Helmut Schmidt” oder noch besser - „Albert Einstein" schreibt, wird der Text für die Literaturgeschichte tauglich. Das bedeutet, dass das Problem, über das ich spreche - das Problem der sogenannten sowjetischen Kultur - nicht nur eines der osteuropäischen Länder ist, sondern, und zwar auf höchst schmerzliche Weise, auch Deutschland betrifft. Wir tabuisieren politisch, ohne es zu bemerken, gewisse Elemente der Sprache und wir bestimmen politisch ihre Unbrauchbarkeit für die Erzeugung ästhetischer Werte. Wir bleiben Menschen unserer Zeit. Aus der längeren historischen 
Perspektive gesehen sind politische Verbote gegen Kunst und Wissenschaft nur vorübergehend.

Ich habe in einem früheren Aufsatz gezeigt, dass in dem - aus heutiger Sicht - Zweifelhaftesten, was wir von der Sowjetzeit geerbt haben, in der stalinistischen Lyrik und Lyroepik der 1940-1950er Jahre, ein renommierter weltliterarischer Code sehr einflussreich die Struktur poetischer Texte organisiert (Undusk 1998: 137-164). Das ist der Code der christlichen Mystik, die Magie der heiligen Namen eingeschlossen. Freilich muss man, um korrekt zu sein, über die antichristliche Magie und Mystik sprechen, weil die Namen, die im Stalinismus als Zauberworte dienten und wirkten, nicht Jesus Christus oder Jungfrau Maria waren, sondern anderer Art: überwiegend Stalin und Lenin, manchmal auch Michail Kalinin oder Viktor Kingissepp, der 1922 erschossene führende estnische Kommunist. Die umweltlichen Realien sind im 20. Jahrhundert naturgemäß andere geworden. Aber im Allgemeinen kann man sich nur darüber wundern, wie gut die Namen Jesus Christus, einerseits, und Stalin/Lenin, andererseits, in alten und neuen Texten miteinander austauschbar sind.

„In Andacht versunken, erwähne ich deinen Namen, / um erleuchtet zu sein in der dunklen Nacht, die mich umgibt [...]". ${ }^{1}$ Dies sind keine Zeilen aus dem mystischen Traktat Die Dunkle Nacht des großen spanischen Mystikers Johannes vom Kreuz aus dem 16. Jahrhundert - nein, obwohl sie auch dort vorkommen könnten. Der Name, der gemeint war, ist Wladimir Lenin, und der Autor ist nicht Johannes vom Kreuz, sondern Johannes Vares (Barbarus), der erste Vorsitzende des Präsidiums des Obersten Sowjets Sowjetestlands im Jahre 1940, dazu ein bekannter Dichter ursprünglich avantgardistischen Profils. Einer der nächsten Vorsitzenden desselben Präsidiums, August Jakobson, ein Schriftsteller auch er, verherrlicht in seinem Nachruf auf Stalin die mystische Omnipräsenz des großen Steuermanns der Menschheit: „Der Name Stalin lebt weiter in unserer umgestalteten Natur - in unseren riesenhaften Wasserbehältern, in unseren immer grüner werdenden Steppen, in unseren rauschenden Wäldern, in unseren goldenen Weizenfeldern [...]”. Und in ähnlichem Ton, der fast den Sonnengesang des heiligen Franziskus in Erinnerung bringt, gibt sich der junge Juhan Smuul seiner Inspiration hin: „Das Land erstreckt sich wie ein Siegeslied, / weit, überreich und schön wie ein Wunder, / mit hohem blauen Himmel. Und das alles / trägt in sich den Namen Stalin." (Poeem Stalinile / Poem an Stalin, 1949). Dies sind Beispiele der hohen stalinistischen Lyrik, und ich werde die Reihe nicht weiterführen, um Sie zu überzeugen: Die alte mystische Tradition weht eigenartig stark über ihnen.

1 Sämtliche Übersetzungen stammen vom Verfasser - J. U. 
UNDUSK

Die stalinistische Mystik hatte ihre seltsame poetische Größe, nur klingt der Name des Gottmenschen falsch. Gute Christen unter uns können solch eine Dichtung im Namen Jesu Christi wohl genießen, Lenin, Stalin und Hitler sind für unseren Geschmack jedoch nicht zu ertragen. Obwohl in der weltlichpantheistischen Naturlyrik ziemlich verbreitet, hatte die mystische Rhetorik in der estnischen kirchlichen Tradition keine stärkeren Wurzeln schlagen können; die Heiligenverehrung ist uns als Protestanten fremd geblieben. Und im heiligen Deportierer Stalin sein Heil zu finden, fiel der Mehrheit der Esten sowieso schwer, obwohl auch seine Macht als imperialer Kriegsführer (der Sieger des Zweiten Weltkriegs, sagte man damals) und Industrialisierer attraktiv war - Macht ist doch immer attraktiv. Dagegen war die russischsprachige Stalinistik zum Teil eine Fortführung der ostkirchlichen Wortgebrauchstraditionen in roter Farbe und damit ein Ort, an dem das Volk seine religiöse Not und Wut legal in neue antichristliche Formen gießen konnte.

Was ich damit sagen will, ist, dass die vom heutigen Standpunkt total falsche Sprache, die wir in der stalinistischen Lyrik vermuten, in einer geschichtlich längeren weltliterarischen Perspektive ihre eigene Tradition finden kann, in der ihre totale Falschheit wenigstens teilweise verschwindet.

Kommen wir zurück zum erzwungenen Sprachgebrauch. Wir entdecken im sowjetischen Textkorpus eine falsche Sprache aufgrund seiner mutmaßlichen Erzwungenheit. Natürlich haben sich manche Autoren - auch um ihre elementare Lebensqualität zu bewahren - gezwungen gefühlt, Gedichte und Romane zu gewissen Themen zu schreiben oder eine gewisse Tendenz oder Rhetorik zu präsentieren, wenn auch später nur durch hauchdünne Nuancen. Ich riskiere jedoch eine These: Die Mehrzahl der literarischen Texte, die zur Sowjetzeit erschienen sind, wurden meines Erachtens subjektiv ehrlich geschrieben. Diese Behauptung ist durch das Gegenargument, dass die Autoren sich ihrer Werke manchmal später auch geschämt haben, nicht so leicht zu widerlegen. Juhan Smuul hat seine Poeme an Stalin nach der offiziellen Verurteilung des Personenkults um den Diktator sehr bedauert, aber nicht, weil er selbst gelogen hat, sondern weil er sich in seinen falschen jugendlichen Idealen und Erwartungen betrogen fühlte. Die Leitfiguren der Lyrikerneuerung zwischen den 1950er und 1960er Jahren, den in Deutschland renommierten Jaan Kross eingeschlossen, haben mitunter die kommunistischen Ideen und die marxistisch-leninistische Weisheit besungen, was um die Zeit der politischen Wende auch peinliche Momente verursachen konnte. Aber nach den Jahren des grenzenlosen staatlichen Terrorismus und den sibirischen Straflagern wirkte das relativ normale Leben in der Sowjetunion der 1960er Jahre eher wie ein Geschenk und der sozialistische Optimismus war nicht vorgetäuscht. 
Der Schriftsteller Paul Kuusberg, einer der besten Romanautoren der 1960er Jahre, war ein aufrichtiger Kommunist bis zum Ende seiner Tage (er starb erst 2003). Kommunist zu sein bedeutete für ihn mehr als der Kommunismus, weil der Letzte nur eine Hypothese war, die die ferne Zukunft betraf, Kommunist konnte man aber schon hier und jetzt in Estland sein - nach Kuusberg war der Kommunist ein humanistisch veranlagter, kluger und ehrlicher Mensch. Kommunist zu sein war sogar wichtiger als die Mitgliedschaft in der Kommunistischen Partei, weil in der Partei nicht unbedingt die sogenannten wahren Kommunisten triumphierten. Aus den „wahren” Kommunisten werden in Kuusbergs Werken oft Außenseiter. Das heißt, Kuusberg nannte seinen positiven Helden einen Kommunisten, was beim heutigen Leser auf der Oberfläche seiner Texte natürlich nur Befremden bewirken mag. Dabei war er aber (weil es ihm als führendem Kommunisten erlaubt war) einer der wichtigsten Entlarver des parteilichen Opportunismus.

Was vom heutigen Standpunkt eine falsche Sprache darstellt, konnte ursprünglich nicht erzwungen, sondern subjektiv echt und richtig sein. Deshalb sollte man, wenn möglich, zwischen zwei Sprachschichten unterscheiden: der einen, die wirklich unter Zwang verwendet und so auch damals gefühlt wurde, und der anderen, die spontan aus den sozialen Diskursen erwuchs, die wir heute zwar als falsche Diskurse werten (z. B. den Diskurs des Kommunismus oder des Nationalsozialismus), die aber damals nicht unbedingt so angesehen wurden. Und die Kehrseite der Medaille gab es auch: Die große Dichterin Marie Under hat im schwedischen Exil estnische Freiheitslieder geschrieben, die zuvor nicht zu den von ihr verfassten Genres gehörten. War sie dazu gezwungen? Ja und nein. Durch den Kontext der verlorenen Heimat - ja. Aber von der falschen Sprache zu sprechen wäre doch unangebracht, weil die Worte der Dichterin persönlich tief empfunden sind.

Einer der beredsamsten Codes der Literatur in totalitären Gesellschaften ist das Schweigen. Die literarischen Werke von Autoren, die ins Exil gegangen sind, wurden tot geschwiegen - bis zu einem gewissen Grad auch die Werke, die sie schon davor geschrieben hatten. Die Eigensinnigsten unter denen, die in der Heimat blieben, schwiegen von selbst oder auf Befehl. In Texten ging man an den politisch wichtigsten Inhalten schweigend oder andeutungsweise vorbei. Solange man Autoren oder Werke beschimpft oder verurteilt („kritisiert“), sind sie noch lebendig. Die gefährlichsten Sachen schweigt man tot. Die „schlechtesten” Autoren wurden nicht dazu gezwungen, eine gewisse Tendenz und Rhetorik zu präsentieren, nein, das wäre schon ein Privileg, ein Angebot gewesen - ihre Stimmen mussten unhörbar sein. Die sowjetische Gesellschaft war wortmagisch veranlagt: Man huldigte bestimmten Namen und Begriffen, man hütete sich peinlich vor anderen. Es galt die Regel: Sprechen ist Freilassen. 
UNDUSK

Um etwas wirklich zu vernichten, musste man darüber schweigen. Verurteilung war schon (oder wieder) eine primitive Form der Anerkennung.

Der stalinistische Personenkult wurde auf dem XX. Parteitag der KPdSU 1956 verurteilt, das hieß übrigens, dass der Name Stalins noch öffentlich genannt werden durfte und nicht verheimlicht worden war. Kurz danach wurde der einst magische Führername völlig verboten, seine öffentliche Erwähnung glich einer politischen Provokation. Genauer gesagt, dies konnte nur im Kontext der Entlarvung des Personenkults geschehen. Oder ein Beispiel aus der estnischen Literatur: Das gut gebaute Propagandastück von Egon Rannet aus der Tauwetter-Periode, Der verlorene Sohn (estn. Kadunud poeg, 1958; deutsch 1959), wurde in mehr als 200 Theatern in der Sowjetunion, der DDR, der Tschechoslowakei, Polen usw. gespielt. Die Vertreter der „feindlichen” westlichen Ideologie sprechen im Stück über die freie Estnische Republik, über studentische Korporationen und sibirische Straflager und singen darüber hinaus die finnische Militärhymne, um sich an den siegreichen Krieg gegen die Russen zu erinnern. Das alles geschieht natürlich in einem scharf kritischen, das ideologische Verbrechen enthüllenden Kontext, doch wird das auf der Bühne dem Publikum sozusagen körperlich vorgespielt: Der Name des Präsidenten der Estnischen Republik wird erwähnt und sogar die einstigen Nationalfarben gezeigt, man hört die Melodie und den Text eines verbotenen Lieds usw. In den späten 1960er und in den 1970er Jahren wäre das schon - auch in kritischem Kontext - unmöglich gewesen: Die Realität, die sich in diesem Stück noch verzerrt widerspiegelt, wurde aus dem öffentlichen Gedächtnis gestrichen, verschwiegen. Die sogenannte Normalisierung war in diesem Fall nur der Schein, der trügt - eine Normalisierung durch das Totschweigen.

Glasnost oder die Kehre an die Öffentlichkeit, geprägt von Michail Gorbatschow in der zweiten Hälfte der 1980er Jahre, begann mit der erneuten Beschimpfung der Dinge, die inzwischen totgeschwiegen waren. Wiederbeschimpfen bedeutete ins Leben rufen. Die Tatsache vergisst man nur zu gerne.

Es gab paradoxe Angelegenheiten. Je mehr Russen in Estland angesiedelt wurden, desto enger wurde das Anwendungsgebiet des russischen Nationennamens - „der Russe” - definiert. Wie man im politischen Slang statt von „Negern” heute von der „schwarzhäutigen Bevölkerung” spricht, musste man anstatt von „Russen” von der „russischsprachigen Bevölkerungsschicht” sprechen. Der Name „Russe” begann sich die Konnotationen anzueignen, die in vielen Sprachen tatsächlich dem „Neger” eigen sind: Man behandelte ihn dabei offiziell! - fast wie ein Schimpfwort, wie einen schrecklichen Tabunamen, an dessen Stelle man nur beschönigende Umschreibungen, Euphemismen, gebrauchen durfte. Aber wie gesagt, solange man einen beschimpft, ist er noch 
am Leben. Nach der Beschimpfung kommt das tötende Schweigen. Und gerade die Russen waren die ersten, die ihre Nationalität - im positiven Sinne - in der Sowjetunion zu verlieren begannen.

Estland war voller Russen, aber in der estnischen Literatur offenbarte sich diese sich immer mehr aufdrängende Anwesenheit nicht, ganz im Gegenteil, die Russen wurden aus der Literatur immer mehr hinausgedrängt. Wem die sowjetestnische Literatur als soziologische Basis für die Untersuchung der estnisch-russischen Beziehungen dient, muss vorsichtig sein: Die Realität und die Literatur stehen umgekehrt proportional zueinander. Es kommen nur einzelne russische Figuren vor und diese sind überwiegend klischeehaft, weil es schwer, sogar unerwünscht oder manchmal gefährlich war, aus einem Russen, aus einem wirklichen Iwanow, in der estnischen Literatur einen vollblütigen Charakter anzufertigen. Ein menschlich komplizierter Russe lag in der Kompetenz der russischsprachigen Literatur. „Die kleinen klugen Augen des Kommissars” ist ein Beispiel des positiven Klischees für das russischsowjetische Militär in der estnischen Literatur: manchmal ohne Nation und ohne Namen, doch allen verständlich. Nicht einfach der Nationenname, sondern bereits ein russischer Familienname konnte politisch provokative Situationen heraufbeschwören. Natürlich gab es den inneren Widerstand gegen alles Russische, die die übersetzte Literatur und Filme reichlich anboten. Die paradoxe Tatsache war jedoch, da $\beta$ auch auf der offiziellen Seite das nackt Russische gefürchtet wurde. Wenn Anfang der 1990er Jahre ein Roman der Schriftstellerin Maimu Berg mit dem Titel Ma armastasin venelast (Ich liebte einen Russen, 1994; deutsch 1998) erschien, war das schon ein Zeichen für die revolutionären Änderungen des politischen Klimas, die inzwischen stattgefunden hatten. Wir mussten alle zwangsweise die russische Sprache und die damit verbundene progressive Kultur lieben, aber um eine Liebe zu einem Russen in aller Öffentlichkeit verkünden zu dürfen - dafür brauchte man erst den Zusammenbruch der Sowjetunion.

Zum Schluss spreche ich über das sogenannte „Wichtigste” - über den Sozialistischen Realismus. Die Methode des Sozialistischen Realismus ist das grundlegende Kennzeichen aller Literatur, die auf dem Gebiet des sowjetischen Landes geboren ist, so wurde auch ich zu meinen Schul- und Universitätszeiten unterrichtet. Bei der Lektüre moderner estnischer oder aber lettischer, litauischer oder russischer Literatur der 1970-1980er Jahre konnte ich nicht verstehen, warum ausgerechnet diese Texte, die der im Westen erschienenen Literatur so ähnlich waren, sozialistisch-realistisch genannt wurden. Sie waren eher ironisch, zynisch, individualistisch, skeptisch bis pessimistisch, in Litauen oft auch betont romantisch. Die logische Lösung des Problems war, dass der Sozialistische Realismus kein ästhetischer, sondern ein geopolitischer, 
UNDUSK

räumlicher Begriff war. Wieder sind wir an der Räumlichkeit der sowjetischen Kultur angelangt.

Theoretisch hat der Sozialistische Realismus fast alles erlaubt. Ich zitiere aus dem ersten Statut des Sowjetischen Schriftstellerverbandes 1934: „Der sozialistische Realismus sichert dem künstlerischen Schaffen außerordentliche Möglichkeiten in Bezug auf die Entwicklung schöpferischer Initiative und die Wahl mannigfaltiger Formen, Methoden und Genres" (zit. nach Klein 2003: 180).

Und so weiter. Ästhetisch sagt das zugleich alles und nichts. Aber die Doktrin des Sozialistischen Realismus war überhaupt nicht erfunden, um den Künstlern neue Wege des Schaffens zu erleuchten, sondern um den Funktionären ein ideologisches Mittel gegen den unbequemen Bereich der freien Künste zu geben. Ein Mittel zur ideologischen Korrektur oder Säuberung oder Tschistka jeder Art, das heißt keine ästhetische Methode, sondern eine Maßnahme zur Gewalt. Die „dialektische Breite” der Methode ermöglichte vor allem die „dialektische Breite” der Repressionen. Der Sozialistische Realismus war räumlich definiert. Diese Doktrin bedeutete einfach, dass jeder Künstler auf dem Gebiet der Sowjetunion im Prinzip der staatlichen Gewalt unterlag. Heute sind auch die russischen Forscher bereit, zuzugeben, dass es einer Selbsttäuschung gleichkommt, sich über den Sozialistischen Realismus als einer künstlerischen Methode Gedanken zu machen. So schreibt z. B. der Kunsthistoriker Vitali Manin: „Der sozialistische Realismus ist vor allem als ein staatliches System der Verwaltung der Künste entstanden. Er selbst war ein organisches Element des totalitaristischen Systems und kann nur als ein Instrument der Parteipolitik im Bereich der Künste verstanden werden. Den sozialistischen Realismus zu den schöpferischen Methoden zu rechnen, ist im wesentlichen demagogisch." (Manin 2011).

Aber wie hat dieses Instrument funktioniert, wie hat man es ins Werk gesetzt? Vor allem durch die breit angelegten „dialektischen” Kampagnen, die das ganze Feld des künstlerischen Schaffens gedeckt haben, den Weg zu jedem Künstler finden und ihm ideologisch drohen konnten. Die liberale Definition des Sozialistischen Realismus war nur ein Lockmittel für die Künstler, die sich damit als fast freischaffende Menschen fühlen konnten; aber freie Hand war dabei eigentlich nicht den Künstlern, sondern den führenden Kulturbürokraten gelassen, die auf der Basis dieser vielversprechenden Definition politische Netze universellen Umfangs ausgeworfen haben. Geben wir einige Beispiele.

Im Namen des Sozialistischen Realismus lief seit 1936 eine Kampagne gegen den „Formalismus und Naturalismus”. Unter solch einer Flagge konnte man jedes Phänomen der Kunst bekämpfen. Jedermann war bedroht, jeder hatte einen Grund zu fürchten, und das war der große Sinn der Unternehmung. Die Nachwirkung dieser Kampagne hat in Estland gleich nach dem Beginn der 
sowjetischen Okkupation in den Jahren 1940-1941 eingesetzt: „Naturalismus ist tatsächlich nur eine der Erscheinungsformen des Formalismus," schrieb der junge Laaban (Laaban 1941: 161). Damals blieb es weitgehend nur eine These ohne größere kulturpolitische Konsequenzen, die erst nach dem Zweiten Weltkrieg, am Anfang der zweiten sowjetischen Okkupation, die Zerstörung der ganzen ästhetischen Situation verursachte. Schon während der ersten Monate des Jahres 1945 begann mit der ideologischen Gleichsetzung von Naturalismus und Formalismus die sich stetig verstärkende Hexenjagd auf den unbequemen Teil der Menschheit (siehe Jäärats 2012: 51f.).

1949 fuhr man mit dem Kampf gegen den „Kosmopolitismus und Nationalismus” fort. Auch während dieser „dialektischen” Geistesverfolgung konnte jeder Autor, jedes Werk verurteilt werden. Beide, sei er ein nationsbetonter oder ein nicht nationsbetonter Autor, Held oder sein Gegner, waren automatisch schuldig. Besonders beliebt waren öffentliche Schuldbekenntnisse, deren „dialektischer” Aufbau als ein Indiz für die Ehrlichkeit der Selbstanklage dienen sollte. So bekannte der Dichter und Romancier Mart Raud im April 1950 vor der Parteiorganisation des Schriftstellerverbandes, dass er bisher unter dem verderblichen Einfluss sowohl der entschiedenen Nationalisten als auch der Kosmopoliten gestanden habe (Olesk 2003: 502f.). Der erste Sekretär der KP Estlands, Nikolai Karotamm, hat sich auf dem berüchtigten 8. Plenum der Estnischen KP im März 1950 - vor seinem erzwungenen Rücktritt vom Amt - zweier Dinge für schuldig bekannt: der Gutherzigkeit und des Stolzes (Plenum 1998: 2666). Wieder eine gute dialektische Mischung, die übrigens Karotamm - aller Wahrscheinlichkeit nach - sein Leben gerettet hat (er wurde nach Moskau „zur wissenschaftlichen Arbeit” geschickt).

Ich habe vom mystischen Code der sowjetischen Ideologie gesprochen. Die Doktrin des Sozialistischen Realismus war ein glänzendes Produkt desselben. Er wurde aber nicht aufgestellt, um neue Erkenntnisse über die geheimnisvolle Welt zu erwerben. Der typisch mystische, das heißt auf begrifflichen Oxymora (die sogenannten „dialektischen Gegensätze“) beruhende Wortgebrauch wurde im Interesse der öffentlichen Gewalt instrumentalisiert. Wer „Krieg ist Frieden” verkündet und dabei physische Macht hat, wie George Orwell in seinem berühmten Roman 1984 gezeigt hat, der hat wirklich grenzenlose Macht.

\author{
Jaan Undusk \\ jaan@utkk.ee \\ Eesti Teaduste Akadeemia Underi ja Tuglase Kirjanduskeskus \\ Roosikrantsi 6 \\ 10119 Tallinn \\ EESTI
}


UNDUSK

\section{Literaturverzeichnis}

Berg, M. 1998. Ich liebte einen Russen. [Übersetzt von I. Grönholm.] Blieskastel: Gollenstein.

Jäärats, M. 2012. Kodanliku mineviku ja sotsrealistliku tuleviku vahel. Eesti kunstikirjutuse diskursused 1944-1948. Magisterarbeit. Tallinn: Eesti Kunstiakadeemia. [Manuskript im Institut der Kunstwissenschaft der Estnischen Kunstakademie.]

Klein, W. 2003. Realismus/realistisch. - Ästhetische Grundbegriffe. Hg. von K. Barck et al. Bd. 5. Stuttgart/Weimar: Verlag J. B. Metzler, 149-197.

Laaban, I. 1941. Formalism kirjanduses kui sotsialistliku realismi vaenlane. - Eesti Keel ja Kirjandus, 2, 156-162.

Laaban, I. 1997. Sürrealismi perspektiive (1944). - I. Laaban. Marsyase nahk: Tekste tekstidest. Tekste piltidest. Tallinn: Eesti Raamat, 39-46.

Manin, V. 2011 = В. Манин. Реацизм с приставкой “соц" и с приставкой "сюр”. -

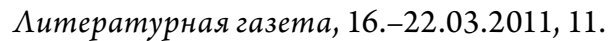

Olesk, S. 2003. Võim kirjanduse kallal: dokument eesti kirjanduse juhtimisest 1950. Võim \& kultuur. Hg. von A. Krikmann, S. Olesk. Tartu: Eesti Kirjandusmuuseum Eesti Kirjandusloo ja Folkloristika Keskus, 481-525.

Plenum 1998 = EK(b)P Keskkomitee VIII pleenumi stenogramm. [Übersetzt von M. Arold, J. Isotamm.] - Akadeemia, 12, 2655-2686.

Rannet, E. 1959. Der verlorene Sohn. Stück in drei Akten. [Übersetzt von G. Jäniche.] Berlin: Henschelverlag.

Undusk, J. 1998. Maagiline müstiline keel. (= oxymora 2). Tallinn: Underi ja Tuglase Kirjanduskeskus.

Undusk, J. 2008. The Fading Reality of Socialist Realism: Aesthetics as a Means of Realpolitik. - Back to Baltic Memory: Lost and Found in Literature 1940-1968. Hg. von E. Eglāja-Kristsone, B. Kalnačs.

Undusk, J. 2013. Sotsialistliku realismi lenduv reaalsus: esteetika kui reaalpoliitika riist. - Vikerkaar, 6, 39-61. 